Informe especial

\title{
La prevención de las enfermedades cardiovasculares: Declaración de Cataluña (continuación)
}

\begin{abstract}
En el último número de esta revista se publicaron en español los tres primeros capítulos del documento The Catalonia Declaration: investing in heart health, donde se presentan abundantes ejemplos de iniciativas para la prevención de las enfermedades cardiovasculares que han producido beneficios de salud apreciables. En las siguientes páginas se presentan sus tres capítulos restantes, que proveen ejemplos adicionales de medidas productivas para la promoción de la salud cardiovascular y describen las barreras que obstaculizan el progreso en este campo, junto con algunas estrategias para superarlas.
\end{abstract}

\section{CONTENIDO}

Capítulo 4

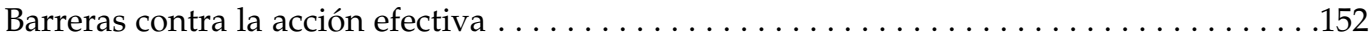

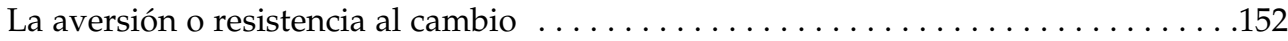

Los lapsos entre la adquisición de los conocimientos científicos y su aplicación . . . .153

El conflicto entre los intereses comerciales y el sector de la salud . . . . . . . . . . 154

La falta de colaboración . . . . . . . . . . . . . . . . . . . . . . . . . . . . 155

Capítulo 5

Estrategias para la inversión en la salud cardiovascular: un marco para la acción . . . . . . .156

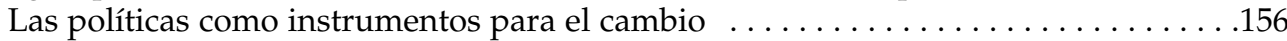

¿Relaciones "descendentes" o "ascendentes"? . . . . . . . . . . . . . . . . . 156

Estrategias para la inversión en la salud cardiovascular . . . . . . . . . . . . . 158

Capítulo 6

Inversiones fructíferas en la salud cardiovascular . . . . . . . . . . . . . . . . . . . 160

La inversión en la salud cardiovascular en el medio laboral . . . . . . . . . . . . . 161

La atención primaria y los programas en entornos clínicos . . . . . . . . . . . . 161

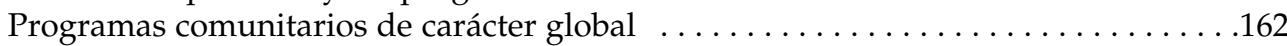

El sector voluntario . . . . . . . . . . . . . . . . . . . . . . . . . . . . . . 164

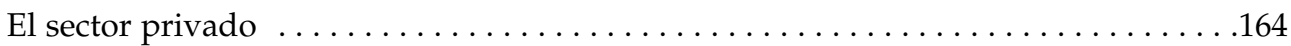

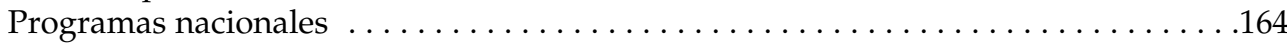

Programas internacionales y regionales $\ldots \ldots \ldots \ldots \ldots \ldots \ldots \ldots \ldots \ldots \ldots \ldots \ldots \ldots \ldots \ldots$

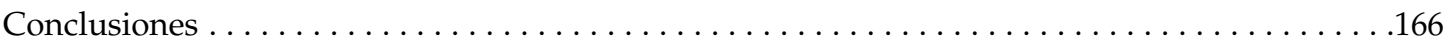

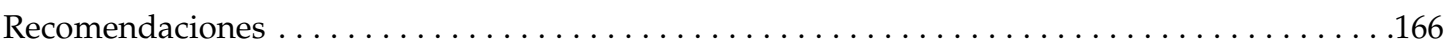

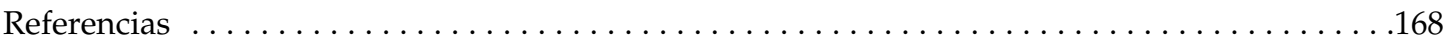




\section{CAPÍTULO 4}

\section{BARRERAS CONTRA LA ACCIÓN EFECTIVA}

Según demuestran los ejemplos recogidos en la Declaración de Cataluña, la prevención de las enfermedades cardiovasculares no solo salva vidas y aminora el sufrimiento que estas ocasionan, sino que puede redundar en un gran ahorro de recursos.

Si las medidas con que se combaten los trastornos cardiovasculares aportan tan notables beneficios para la salud y la economía ¿por qué no se están aplicando de manera eficiente, generalizada y oportuna? ¿Por qué continúa habiendo una epidemia de mortalidad por enfermedades cardiovasculares en los países en desarrollo y en Europa Oriental?

La respuesta radica en las ideas, acciones y conductas de individuos de todos los estratos sociales y de las organizaciones y sistemas a los que están adscritos.

En este capítulo examinaremos las barreras que dificultan el progreso y que explican, al menos en parte, la ausencia de medidas adecuadas en tantos países del mundo. Nos concentraremos en los siguientes factores, que pueden obstaculizar enormemente cualquier adelanto en el campo de la salud cardiovascular:

- La aversión o resistencia al cambio;

- los lapsos entre la adquisición del conocimiento científico y su aplicación;

- los conflictos de intereses con los sectores del comercio y el mercado;

- la falta de colaboración.

Examinaremos la manera en que estos factores determinantes pueden influir en el público y en las instituciones de distintos niveles, en el individuo y la familia, en grupos pequeños y comunidades, en las organizaciones y sistemas $\mathrm{y}$, finalmente, en los gobiernos.

De no hacerse frente a estos obstáculos, los resultados de cualquier intervención serán pobres y limitados y la toma de decisiones se verá frustrada por la ausencia de una colaboración intersectorial.

En este capítulo se presenta cada una de las posibles barreras que hemos mencionado y en el capítulo 5 se examinan algunas estrategias para superarlas.

\section{La aversión o resistencia al cambio}

La tradición - conjunto de normas sociales de cada cultura o subgrupo dentro de un país- puede automáticamente obstaculizar el cambio. Las normas, por definición, son antagónicas a la adopción de innovaciones o tienden a amortiguarlas. Lógicamente, se trata de una resistencia natural. De hecho, la supervivencia del ser humano depende de cierto grado de resistencia a innovaciones autodestructivas. Por ejemplo, para protegerse del envenenamiento, a través de la historia la población ha evitado el consumo de ciertas plantas prohibidas por la creencia popular. No obstante, en ocasiones las costumbres pueden constituir obstáculos que menoscaban la salud, como ilustran estos dos ejemplos:

- Cierta población africana se abstenía de consumir una planta local rica en betacarotenos porque, según la creencia popular, la planta producía diarrea. Una ceguera diseminada que posteriormente se destapó en ese territorio fue causada por una deficiencia de vitamina A que hubiera podido prevenirse con el consumo regular de pequeñas cantidades de la planta.

- El tabaquismo siguió siendo un hábito muy arraigado en las sociedades occidentales, aun después de conocerse sus peligros. Un ejemplo es el de Sir Richard Doll, epidemiólogo británico, que seguía ofreciendo cigarrillos a las personas que lo visitaban mucho tiempo después de su descubrimiento en 1940 de una asociación entre el consumo de cigarrillos y el cáncer de pulmón.
Si bien la tradición puede tener una función protectora, las epidemias requieren una respuesta inmediata. El desafío consiste en alentar a la población en general y a los formuladores de las políticas sanitarias en particular a adoptar innovaciones beneficiosas para la salud. Para fomentar cambios, es preciso superar el pesimismo que tan frecuentemente caracteriza a quienes cambian con lentitud o se resisten al cambio, aun después de haberse constatado los beneficios del mismo para la sociedad. Un proyecto en Portugal (recuadro 29) proporciona un ejemplo de cómo superar la barrera de la tradición.

A pesar del éxito de intervenciones como la portuguesa, se percibe cierto pesimismo en todos los estratos de la sociedad en torno a los beneficios de iniciativas sociales bien planificadas y constructivas. Demasiadas veces los líderes que moldean la opinión pública y los formuladores de políticas simplemente no creen que la acción pueda surtir ningún efecto, ni en forma de programas de educación o capacitación, ni en forma de incentivos.

Este pesimismo podría estar arraigado en el fatalismo. Cuando las medidas de promoción de la salud y de prevención de enfermedades no arrojan beneficios patentes, la resistencia a las innovaciones es una tendencia natural.

Un problema fundamental en estos casos puede ser la ausencia de una sensación de eficacia personal, es decir, de la seguridad, disponibilidad y habilidad autopercibida que se necesitan para efectuar un cambio en particular (Bandura, 1986). Esta limitación puede afectar a las instituciones, así como a sus integrantes, y la situación redunda en una falta de eficacia colectiva. Para tener la sensación de "autoeficacia", es preciso creer que determinado problema es importante y que un cambio sería beneficioso. Las personas que dudan de su propia eficacia y los grupos o instituciones con poca fe en su utilidad colectiva suelen tener menos experiencia con situaciones que exigen cambio y manifiestan un mayor pesimismo que otros en torno a las posibilidades de lograr buenos resul- 
RECUADRO 29. La reducción del consumo de sal en Portugal

En Portugal se estudió el efecto de un programa de educación sanitaria para reducir el consumo de sal y la tensión arterial en dos comunidades rurales pareadas, cada una con una población cercana a los 800 habitantes. Ambas comunidades se seleccionaron por su alto consumo de sal, su elevada prevalencia de hipertensión y su alto grado de concienciación pública sobre la carga representada por los trastornos de la tensión arterial y los accidentes cerebrovasculares. La comunidad donde se efectuó la intervención se eligió porque en ella era más fácil comunicarse con los líderes locales que en la aldea que sirvió de control. La estrategia de la intervención se centró exclusivamente en la educación sanitaria. Se pidió a los habitantes de la zona de intervención 1) que cocinaran con menos sal y que la sustituyeran con hierbas aromáticas y otros condimentos, 2) que consumieran menos bacalao y embutidos, que en la cocina portuguesa se elaboran con mucha sal, y 3) que prepararan el pan con menos sal. La intervención tuvo el apoyo entusiasta de los líderes, médicos y miembros del personal de enfermería de la comunidad. El equipo de investigadores visitó con regularidad la comunidad en que se llevó a cabo la intervención con el fin de dar charlas ante grupos y de enseñar a los habitantes a cocinar con menos sal. También se organizaron concursos destinados a fomentar el cultivo casero de hierbas aromáticas y su uso en la cocina.

Al principio el consumo de sal era muy grande (aproximadamente $360 \mathrm{mmol} /$ persona/día) y $30 \%$ de la población era hipertensa (tensión arterial diastólica $>95 \mathrm{mmHg}$ ). Después de un año, tanto las encuestas alimentarias como las muestras de orina indicaron que en la comunidad donde se había realizado la intervención el consumo de sal había disminuido en $50 \%$ y la tensión arterial promedio había bajado 3,6/5,1 mmHg. Al cabo de 2 años, esta había bajado $5,0 / 5,1 \mathrm{mmHg}$. En la comunidad que sirvió de control las tensiones diastólicas permanecieron inalteradas y las sistólicas aumentaron. La diferencia de tendencias fue muy significativa. Se considera que una reducción de 5,0 $\mathrm{mmHg}$ en la tensión arterial redundaría en un descenso de $25 \%$ en la frecuencia de hipertensión y que ello a su vez contribuiría a una notable reducción de las enfermedades cardiovasculares.

Fuente: J. Forte, et al., 1989
Por añadidura, los encargados de asegurar el cumplimiento de las metas de salud no están en obligación de rendir cuentas. Frecuentemente, la propia maquinaria decisoria fomenta en los funcionarios electos una actitud pasiva y reaccionaria, en lugar de despertar en ellos tendencias innovadoras conducentes a beneficios perdurables y a una inversión genuinamente rentable en la salud.

En general, los formuladores de las políticas de salud se resisten al cambio, quizá en parte por su falta de experiencia personal en materia de prevención o por la inercia generada por la presión de intereses comerciales, pero también por su afición general a las soluciones únicas y sencillas. La tendencia a asignar fondos para la curación de las enfermedades en vez de su prevención obedece en parte a una necesidad humana muy patente: los enfermos necesitan atención inmediata. Pero esta falta de financiamiento también se puede atribuir a la frecuente falta de apreciación de los beneficios de la prevención en el largo plazo, ya sean económicos o humanos. La Declaración de Cataluña intentará persuadir a los funcionarios del nivel normativo, con el respaldo de ejemplos convincentes, de que las medidas preventivas que se aplican sostenidamente resultan efectivas en función del costo.

La necesidad de reparar en el futuro menos inmediato es más apremiante en el área de la salud cardiovascular que en otras áreas de la salud pública simplemente porque la planificación y asignación de fondos tienen que hacerse con tanta anticipación. Con frecuencia se torna necesario formar alianzas entre los diferentes sectores del gobierno y adoptar formas de obrar completamente nuevas.

No cabe duda alguna de que la aceptación de responsabilidad es importante; en algunos países este aspecto ha evolucionado más que en otros. Dos ejemplos de la incorporación de responsabilidad dentro del proceso de planificación están dados por los objetivos del proyecto estadounidense Healthy People 2000 y el plan de salud 
de Cataluña (recuadros 30 y 31). En ambos casos, los problemas se han documentado sistemáticamente, los objetivos se han establecido de manera clara, se dispone de una estructura para comprobar el cumplimiento de las metas y los funcionarios electos y no electos rinden cuentas ante sus respectivos organismos legislativos.

A la hora de aplicar los conocimientos científicos a la resolución de los problemas relacionados con la salud cardiovascular, es preciso tener en cuenta los problemas sanitarios específicos de cada lugar, porque los factores de riesgo varían enormemente en distintas partes del mundo. Por lo tanto, si se cuenta con ayuda internacional para cerrar la brecha temporal entre la adquisición de los conocimientos científicos y su aplicación, aquella deberá encaminarse primero hacia la evaluación de los factores de riesgo, especialmente en países en desarrollo.

\section{El conflicto entre los intereses comerciales y el sector de la salud}

Los países, independientemente de su grado de desarrollo económico, se ven sometidos a la presión de intereses que pueden frustrar la adopción de políticas para la promoción de la salud. Por ejemplo, la necesidad de disponer de fuentes de empleo en industrias que contaminan el ambiente pugna con la necesidad de aire puro. Asimismo, la protección de las prácticas agrícolas tradicionales puede ser ventajosa desde el punto de vista político, aun cuando los productos son nocivos para la salud, como en el caso del cigarrillo. Los subsidios pueden llevarse a extremos que violan la lógica y demasiadas veces los beneficios inmediatos superan a los obtenidos en el largo plazo. Por ejemplo, un estudio sobre políticas que se llevó a cabo para la Comunidad Económica Europea (recuadro 32) reveló que los subsidios de la industria agrícola suelen estimular la producción a gran escala de productos lácteos, huevos, carne de cerdo y carne de res, y el resultado es un consumo excesivo de colesterol y grasas saturadas.
RECUADRO 30. Responsabilidad del Ministro de Salud de Cataluña por el plan de salud

Cataluña, como otras comunidades autónomas de España, tiene un plan de salud que se formuló a partir de la Ley General de Sanidad del Conjunto del Estado y la Ley de Ordenación Sanitaria de Cataluña. Este ordenamiento jurídico, originalmente preparado por el Departamento de Sanidad, tuvo la fuerte participación de los profesionales de la salud y de la población en general. Aproximadamente 4000 catalanes participaron en la redacción del documento final.

El plan fue aprobado por el Gobierno Autónomo de Cataluña en 1994 y sometido al Parlamento. Aunque la ley no exigía su aprobación parlamentaria, al solicitar su presentación ante esa cámara y el respaldo del plan, el Ministro de Salud se comprometió públicamente a alcanzar los objetivos del plan y así asumió el deber de rendir cuentas ante el Parlamento y los ciudadanos por los éxitos y fracasos del plan.

Distintas variantes de este enfoque, apoyadas por las leyes nacionales o regionales, están en desarrollo en varias regiones autónomas de España. El proceso de involucrar a muchas personas en el diseño de un plan de salud representa un avance en el desarrollo de políticas para la promoción de la salud y la prevención de enfermedades. Por añadidura, el compromiso institucional es útil no solo porque obliga a los formuladores de políticas a elaborar propuestas racionales y factibles, sino porque establece el antecedente de hacer que los individuos se responsabilicen por el cumplimiento de las políticas una vez que han sido aprobadas.

Fuentes: Ll. Salleras, et al., 1994; Department de Sanitat i Seguretat Social de la Generalitat de Catalunya, 1993.
Simultáneamente, los países con muy poco capital y los que están en proceso de transición hacia economías de mercado parecen destinados a ser víctimas de la industria tabacalera, que representa un perfecto ejemplo del intercambio de mercantilismo y lucro por un lado, y enfermedad y muerte por el otro, en el ámbito mundial. En el caso del tabaco, la extraordinaria ren- tabilidad de las compañías tabacaleras transnacionales les ha permitido en años recientes ampliar aceleradamente su mercado en diferentes partes del mundo, por medio de la compra y renovación de compañías tabacaleras talaciones nuevas para alcanzar mayor eficiencia en la elaboración de cigarrillos. Sumada a la intensa propaganda ya existentes o de la edificación de ins-

\section{RECUADRO 31. Healthy People 2000}

Healthy People 2000, iniciativa de prevención de los Estados Unidos, fue lanzada por el Servicio de Salud Pública del país en 1990. El documento presenta 300 objetivos específicos y mensurables que pueden alcanzarse para el año 2000 en 22 áreas prioritarias relacionadas con la promoción y protección de la salud y con los servicios clínicos de prevención. Estos objetivos se consideran de importancia vital si se han de alcanzar tres metas nacionales de carácter general: mejorar la calidad de la vida, reducir las disparidades que existen en el estado de salud de distintos grupos de población, y lograr el acceso de todos los habitantes del mundo a servicios de atención primaria y de prevención.

Healthy People 2000 se diseñó intencionadamente como programa nacional y no federal. Si bien es cierto que las agencias federales del Servicio de Salud Pública de los Estados Unidos son las principales encargadas de las 22 áreas prioritarias de la salud que requieren mejoría, el sector no gubernamental ha asumido la principal responsabilidad de dirigir el desarrollo y cumplimiento de los objetivos. Un consorcio integrado por alrededor de 300 organizaciones y agencias no federales sirvió de comité directivo durante los 3 años que tardó el proceso de definir los objetivos y desde entonces $85 \%$ de los estados (y muchas municipalidades) han establecido sus propias metas y objetivos según las necesidades de sus poblaciones y las condiciones en que viven. Cada mes se evalúa cuánto se ha avanzado hacia el logro de los objetivos, anualmente se provee un resumen actualizado en la publicación estadística Health United States, y en el intermedio se hace una revisión y se indican las correciones necesarias a mitad de la década. Healthy People 2000 ha dado grandes resultados como vehículo para señalar prioridades y reclutar participantes.

Fuente: U.S. Public Health Service 1991; 1995. 


\section{RECUADRO 32. Política Agraria Común de la Comunidad Económica Europea}

Durante la década de 1990, la Política Agraria Común (PAC) que rige en toda la Comunidad Económica Europea (CEE) ha llevado a un análisis de los factores que influyen en la producción de alimentos. Según esta Política, la producción primaria está determinada por el precio más que por ningún otro factor, incluidas la nutrición y la salud. El contenido graso de las carnes de res y cordero no se está reduciendo porque los productores carecen de un incentivo financiero para eliminar la grasa. La PAC está investigando maneras de subsidiar la producción de alimentos lácteos con un menor contenido de lípidos. Aunque dicha entidad ha tenido ciertos triunfos en la promoción de una producción de alimentos más sanos, en general la experiencia ha demostrado que la presión política de las poderosas industrias de la carne y los productos lácteos no ha permitido a la CEE dedicarse de lleno a establecer políticas agrarias beneficiosas para la salud cardiovascular.

Fuente: L. Stockley, 1993. que reciben las marcas locales de mayor popularidad, esta expansión comercial permite a las tabacaleras ejercer una gran presión política en directa oposición a las medidas promulgadas por el sector de la salud (recuadro 33).

Encontrar maneras de contrarrestar las maniobras de las compañías tabacaleras representa un verdadero desafío, en lo referente a las políticas, para cualquier entidad interesada en la salud cardiovascular. El ejercicio de presión política debe complementarse con otras estrategias antitabáquicas, que pueden adaptarse de países donde han dado buenos resultados. Ante todo, los promulgantes deberán ofrecer argumentos sostenidos, pertinentes y económicamente convincentes encaminados a demostrar que cuando un país obstaculiza la producción y comercialización del tabaco, está haciendo inversiones rentables en la

\section{RECUADRO 33. La invasión de Europa Oriental por las compañías tabacaleras}

La caída de la cortina de hierro abrió la puerta para capturar el lucrativo mercado del cigarrillo de tipo norteamericano. Debido a la gran demanda de productos estadounidenses que existía en ese momento, la comercialización estratégica de un tabaco más suave, como el que se usa en este tipo de cigarrillos, desplazaría a las marcas distribuidas por las compañías de propiedad estatal y difundiría enormemente el consumo de cigarrillos. Un ejemplo claro de esta táctica de mercadeo se produjo en 1992, cuando la compañía tabacalera Phillip Morris de los Estados Unidos compró la empresa Tabac, propiedad del gobierno de la antigua Checoslovaquia. La Phillip Morris hizo una oferta que superó en US\$ 100 millones a la de sus principales competidores en una compra que costó US\$400 millones. Para justificar la compra, la compañía adujo que la inversión proporcionaba una espléndida oportunidad para entrar en el enorme mercado que estaba aún sin explotar en Europa Oriental, especialmente en Rusia.

Aunque otras compañías tabacaleras transnacionales también planean invertir o ya han invertido grandes capitales, la Phillip Morris sola espera gastar $\$ 1000$ millones en mercadeo en Europa Oriental, porque estiman que actualmente se venden 700000 cajetillas de cigarrillos al año o $40 \%$ más que el volumen de ventas anual dentro de los Estados Unidos. A la luz de esta cifra redonda, Andreas Gembler, jefe de operaciones de la Phillip Morris en ese territorio, declaró que "No se puede pasar por alto al consumidor que vive en esta parte del mundo".

Aunque los gobiernos de esa región encuentran halagadora la inversión extranjera, el sector sanitario ha expresado inquietud por las consecuencias que ella pueda acarrear. Por ejemplo, en una carta enviada en septiembre de 1993 al presidente Clinton, los funcionarios de la salud pública de 13 países pertenecientes al antiguo bloque soviético, incluida la República Checa, declararon que: "Las empresas tabacaleras transitorias explotan la urgente necesidad de nuestros países de invertir capital de manera inmediata y en tal proceso menoscaban nuestra salud y arruinan nuestro bienestar económico". A pesar de esta resistencia, los incentivos económicos que proporcionan esas compañías han tenido poca oposición jurídica.

Fuente: Chicago Tribune, 1993. salud (Max y Rice, 1995; Warner y Fulton, 1995).

Pueden citarse varios triunfos. Se ha demostrado en muchos países industrializados que mediante un activismo político sistemático y organizado, los profesionales de la salud han podido contrarrestar la presión comercial de los cabildos de la industria tabacalera. La Declaración de Victoria afirma, además, que la buena salud cardiovascular puede favorecer la actividad comercial y que esta, a su vez, puede fomentar la salud cardiovascular. También se pueden señalar muchos otros ejemplos de colaboración productiva entre los defensores de la salud pública y los representantes de los intereses comerciales del sector privado. Esta colaboración es absolutamente fundamental para poder aplicar las políticas de salud intersectoriales que se recomiendan en esta Declaración.

Por razones prácticas, se aconseja adiestrar a los grupos dedicados a la promoción de la salud en el uso de técnicas para contrarrestar la presión política que ejercen los defensores de los grandes intereses comerciales. En muchos países, la industria del tabaco, el sector agrícola y la industria alimentaria disponen de mecanismos de presión política bien financiados que destinan gran cantidad de recursos a tratar de ejercer influencia sobre los ministerios y las cámaras legislativas. Dicha influencia puede combatirse mediante la formación de grupos defensores, sobre todo si estos logran que los propios funcionarios públicos y los políticos de mayor relieve adopten hábitos de vida saludables.

Es más fácil, por ejemplo, convencer a los funcionarios electos de la necesidad de tener aire puro dentro de los edificios cuando aquellos no son fumadores.

\section{La falta de colaboración}

La colaboración entre instituciones y dependencias burocráticas obviamente tiene valor para la protección de la salud cardiovascular y cada vez se notifican más ejemplos de colaboracio- 
nes productivas, especialmente en el nivel de la comunidad.

En los niveles más altos del gobierno central, sin embargo, a menudo se observa una falta de colaboración. Dentro del sistema sanitario en particular, los problemas de jurisdicción pueden dificultar una colaboración fructífera con otras instituciones o dependencias.

De especial importancia son las divergencias ideológicas en torno a la prevención, que en ocasiones han frustrado la colaboración entre grupos interesados en la promoción de la salud y los responsables de la atención sanitaria. Estas diferencias obedecen principalmente a distintos puntos de vista sobre la importancia de los problemas prevenibles de menor urgencia cuando existen otros que requieren atención con mayor prontitud. De no percibirse una amenaza inmediata para la salud, no se considera perentorio obrar con rapidez. Se permite que persistan problemas de salud que podrían prevenirse, mientras que las acciones se dirigen hacia la atención de trastornos claramente sintomáticos cuyo diagnóstico y tratamiento requieren tecnologías complejas y un uso intenso de servicios asistenciales.

Ninguna de las conductas que suelen aumentar el riesgo de enfermedad coronaria es inalterable, especialmente mientras más surgen ejemplos de inversiones rentables en la salud cardiovascular. Como se afirma en la recomendación 5 de esta Declaración, las escuelas y dependencias del gobierno responsables de la formación de los profesionales de la salud deberán proporcionar incentivos para la promoción de investigaciones sobre la transferencia de tecnologías, con la meta de desarrollar, poner a prueba y difundir métodos de intervención efectivos en función del costo contra los factores de riesgo de las enfermedades cardiovasculares.

\section{CAPÍTULO 5}

\section{ESTRATEGIAS PARA LA INVERSIÓN EN LA SALUD CARDIOVASCULAR: UN MARCO PARA LA ACCIÓN}

Jamás hay que dudar de que unos cuantos individuos tesoneros pueden cambiar el mundo. De hecho, ha sido siempre la única manera en que las cosas han cambiado.

Margaret Mead (la eminente antropóloga, ya fallecida)

\section{Las políticas como instrumentos para el cambio}

En el llamamiento a la acción de la Declaración de Victoria, se pidió a las coaliciones y alianzas que llevaran a cabo procesos de cambio y de implantación de políticas mediante intervenciones en todos los niveles gubernamentales, aprovechando en su totalidad los recursos a su alcance.

En la Declaración de Cataluña se proporciona información adicional para ayudar a los países y a sus debidas instituciones, coaliciones y agencias del gobierno a poner en práctica políticas conducentes a la creación de planes de acción aplicables en cualquier parte del mundo.

El desarrollo de políticas sanitarias sobre asuntos de mayor o menor trascendencia ofrece la oportunidad de aprovechar el poder de la sinergia, puesto que las alianzas confieren al todo un valor mayor que el de las partes. Sean oficiales $\mathrm{u}$ oficiosas, tales alianzas permiten coordinar mejor las actividades, compartir recursos y proveer apoyo técnico a otras entidades. Asimismo, las alianzas y coaliciones también mejoran la capacidad de difundir información y de obtener apoyo político.

Para aplicar las políticas adecuadamente es necesario establecer nexos interactivos, en lo tocante a políticas y recursos, entre organismos gubernamentales y no gubernamentales de los tres niveles: el internacional y regional, el nacional y el local, que incluye la población en general y los grupos comunitarios. En capítulos anteriores se presentaron ejemplos de programas fructíferos en que se ilustraban estos nexos, su interdependencia y su refuerzo mutuo. Los nexos se aplican en materia de políticas, recursos disponibles, intercambio de información y vigilancia del progreso de los planes de acción.

\section{¿Relaciones "descendentes" o "ascendentes"?}

FIGURA 5. Funciones relativas de la educación y la regulación en los diferentes niveles de gobierno
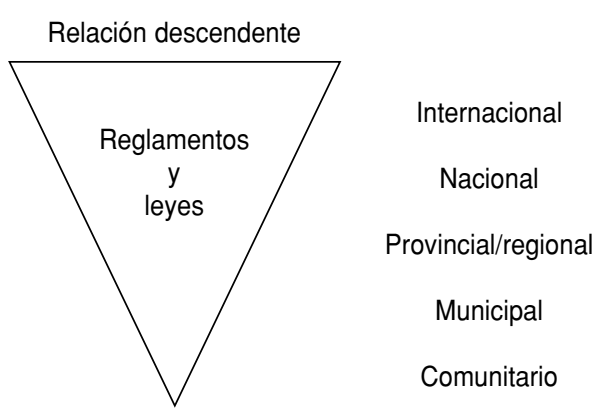

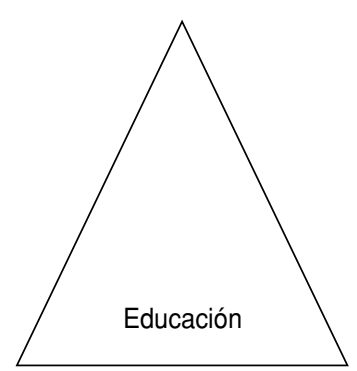

Relación ascendente
¿Cómo difieren las relaciones y funciones de los diversos niveles de organización, desde el internacional hasta el de grupos comunitarios locales? Cabe afirmar, en términos generales, que los niveles superiores del gobierno, en virtud de su relación vertical o "descendente" con respecto al sector de la salud y a otros sectores sociales (figura 5), pueden ser buenas fuentes de financiamiento, de recomendaciones políticas y de la adopción de medidas para la regulación 
de impuestos, transportes, medio ambiente, educación, recreación, responsabilidad fiscal y otras áreas. También pueden aportar la masa crítica de investigadores nacionales y regionales para actividades de investigación y servir de vínculo con las fuentes de recursos en el ámbito internacional.

Sucede, por el contrario, que a medida que se abandona el nivel comunitario y se asciende progresivamente (figura 5) hasta llegar a las esferas sociales, como la salud, el medio principal para lograr cambios en la salud pública radica en procesos educativos que brindan información, imparten habilidades y activan a la población. Estas relaciones descendentes y ascendentes se advierten en todos los ejemplos descritos en capítulos anteriores.

Cuando el público y los grupos comunitarios, entre ellos los de activismo social, están dotados de suficiente información, los hechos pueden respaldarlos a la hora de aplicar la presión política necesaria para ejercer influencia sobre las acciones del gobierno. Esto se constató en el notable ejemplo de la batalla política que llevó a la aprobación del impuesto sobre los productos del tabaco en California (recuadro 34).

En la década actual existen mayores oportunidades de iniciar intervenciones de tipo descendente, gracias a los conocimientos adquiridos durante los diversos proyectos de demostración realizados en distintas partes del mundo. Esta estrategia se apoya en gran medida en el desarrollo de políticas para generar consenso en los niveles internacional, nacional y local, y para consolidar alianzas que promuevan la colaboración en los distintos niveles.

La Iniciativa Canadiense de Salud Cardiovascular (capítulo 2) constituye un ejemplo de una estrategia descendente que brindó a grupos ciudadanos y defensores de la salud pública la oportunidad de actuar. En este caso, el ímpetu organizador originalmente provino de un notable grupo de funcionarios de los departamentos de salud federales y provinciales, apoyados por el asesoramiento y respaldo de algunos científicos del campo de la medicina y de autoridades de la salud

\section{RECUADRO 34. La batalla política para lograr la gravación del cigarrillo en California}

La Proposición 99 de California (véase el recuadro 14 en el capítulo 3) fue aprobada por iniciativa de agencias sanitarias, organizaciones médicas y grupos defensores del ambiente, tras una lucha mancomunada que duró 2 años. Entre las actividades llevadas a cabo en esta campaña figuran la realización de encuestas, la formulación de planes estratégicos, la obtención de cobertura por los medios de comunicación y el mantenimiento de la presión necesaria no solo para llevar la medida propuesta a votación, sino también para lograr que se adoptara.

La coalición, que a propósito se mantuvo alejada de la legislatura estatal donde los intereses tabacaleros tenían gran influencia, apeló directamente al público por los medios locales, tan numerosos que las compañías tabacaleras no pudieron silenciarlos. En ese foro abierto, los promulgantes aprovecharon la inquietud del público con respecto al tabaco y a la juventud, así como la poca credibilidad de las tabacaleras ante el público.

En la fase inicial de la campaña, las encuestas efectuadas por la propia industria demostraron que esta no podía minar el argumento central de la coalición, es decir, que la gravación de los productos del tabaco redundaría en un beneficio para la salud. Para restar atención a tan claro argumento, varios meses antes de la elección la industria tabacalera se dedicó a inundar la televisión, la radio y los medios de comunicación impresos con mensajes propagandísticos encaminados a demostrar que la gravación era regresiva y que afectaría principalmente a personas de la clase obrera.

La coalición defensora de la gravación del tabaco tenía recursos limitados con que responder debidamente. Valiéndose de la red comunitaria que había establecido durante la campaña en que se recolectaron las firmas necesarias para llevar la medida a una votación, la coalición dependió en gran parte de la cobertura gratuita por los medios propagandísticos. La iniciativa tuvo el apoyo entusiasta de los mismos, como demuestra el hecho de que solo tres de los varios centenares de periódicos en el estado publicaron editoriales en contra de la campaña.

Como último recurso, la industria tabacalera demandó legalmente a la coalición. No solo perdió el litigio, sino que la publicidad generada por el caso incrementó la popularidad de la iniciativa.

Llegado el día de la elección, la industria del tabaco había gastado cerca de US\$21,4 millones, mientras que la coalición había gastado solamente US\$ 1,6 millones. No obstante esta gigantesca desventaja económica, la coalición salió victoriosa: el 8 de noviembre de 1988, el público votante de California aprobó la Proposición 99 con un voto mayoritario de 58\%.

Las organizaciones que buscan aumentar el impuesto sobre los productos del tabaco, como la Proposición 99, deben estar preparadas para tomar medidas adicionales, más allá de montar una campaña agresiva, para lograr la aprobación de la iniciativa frente a la oposición de las tabacaleras. Las campañas a favor de gravar el cigarrillo también deben prepararse, como hizo la coalición en California, para proteger el programa en la legislatura una vez que la medida haya sido aprobada por votación pública.

Fuente: M. Traynor y S. Glantz, 1995.

pública con poder de persuasión. La iniciativa consistió en la asignación de recursos financieros para montar en toda la provincia proyectos de demostración que pusieran a prueba la capacidad de movilización de la comunidad en torno a la prevención de las enfermedades cardiovasculares. Este uso acertado de los fondos procedentes de un nivel "superior" ha llevado a la formación de alianzas eficaces entre el Ministerio de Salud del Canadá, funcionarios de la salud pública en el nivel provincial, organismos de asistencia voluntaria e investigadores afiliados a universidades.

Un segundo ejemplo es el proyecto Salud de la Nación del Reino Unido, que fue iniciado por el gobierno con el apoyo de grupos defensores de la salud pública y fundaciones privadas. Este proyecto, procedente del nivel superior, acaba de poner en marcha un gran número de programas para la prevención de las enfermedades cardiovasculares y de otros problemas importantes de salud (recuadro 35).

Raras veces se producen programas nacionales fructíferos sin alguna demostración previa de buenos resultados en una zona del país. Heartbeat Wales, programa global que se instituyó en todo el principado de Gales, demostró claramente que dentro de una unidad gubernamental autónoma se pueden formar alianzas productivas 


\section{RECUADRO 35. Reino Unido: Salud de la Nación}

En 1992, el gobierno del Reino Unido decretó que la promoción de la salud era objetivo central de sus políticas y estableció diversas metas nacionales en el campo de la prevención de las enfermedades cardiovasculares. El documento Health of the Nation White Paper, que se elaboró después de un largo proceso de consultoría, provee un marco de acción para todos los sectores en los niveles nacional y local. El gobierno coordina las acciones por mediación de un subcomité en el gabinete y el Departamento de Salud coordina la labor en todos los departamentos gubernamentales y monitorea el progreso hacia el logro de las metas en el plano nacional. El Departamento de Salud también trabaja con la Dirección de Educación Sanitaria, organización sin fines de lucro cuyos fondos provienen del Departamento de Salud, de otros departamentos del gobierno, del sector privado y de organismos internacionales. A la Dirección de Educación Sanitaria se le ha delegado la responsabilidad de montar grandes programas preventivos nacionales en áreas como el control del tabaquismo, la promoción del ejercicio físico y los buenos hábitos alimentarios. Con este fin, la organización está emprendiendo actividades de investigación extensas para estudiar la efectividad de los programas de promoción de la salud y de algunos aspectos del proyecto Salud de la Nación.

Fuente: P. Lincoln, comunicación personal, 1995.
- trabajar con el sector voluntario y montar programas de capacitación para ayudar a los profesionales en su labor con los miembros de la comunidad;

- trabajar con las organizaciones cívicas y religiosas existentes;

- reorientar el sistema de atención sanitaria hacia la prevención;

- utilizar el sistema educativo existente, desde las guarderías infantiles hasta la educación del adulto en todas sus etapas, y llegar a los niños por medio de las escuelas, la familia y los medios de información;

- establecer contacto con la población en los lugares de trabajo;

- formar alianzas con el sector privado;

- trabajar con los medios de comunicación (locales, regionales y nacionales);

- educar al público en general para que aprenda a exigir un ambiente y una alimentación más sanos (mediante el poder de la opinión pública);

- educar a los formuladores de políticas, líderes de opinión y profesionales de la salud (incluidos los que trabajan en el campo de la salud pública) para que provean oportunidades de acción.

Dentro del marco de acción elegido, que puede ser de carácter ascendente, descendente, o mixto, el paso siguiente es aplicar un modelo gerencial eficiente a la tarea de diseñar un programa para cualquier nivel, bien sea una iniciativa de alcance nacional o un programa aplicado en el medio escolar o laboral.

En la sección siguiente de este capítulo se describe un modelo para la generación de cambios que comprende ocho etapas: identificar el problema; redoblar el compromiso; desarrollar y perfeccionar los métodos; movilizar recursos; emprender acciones; evaluar los resultados, difundirlos, y reciclar, siempre que sea necesario (figura 6). Estos pasos, adaptados de un modelo diseñado por un equipo de investigadores expertos en la aplicación de programas de promoción de la salud globales, integrados y comunitarios (Farquhar, et al., 1991), ayuda- 
FIGURA 6. Secuencia idealizada de planificación y gestión para lograr inversiones eficientes $y$ efectivas en la salud cardiovascular

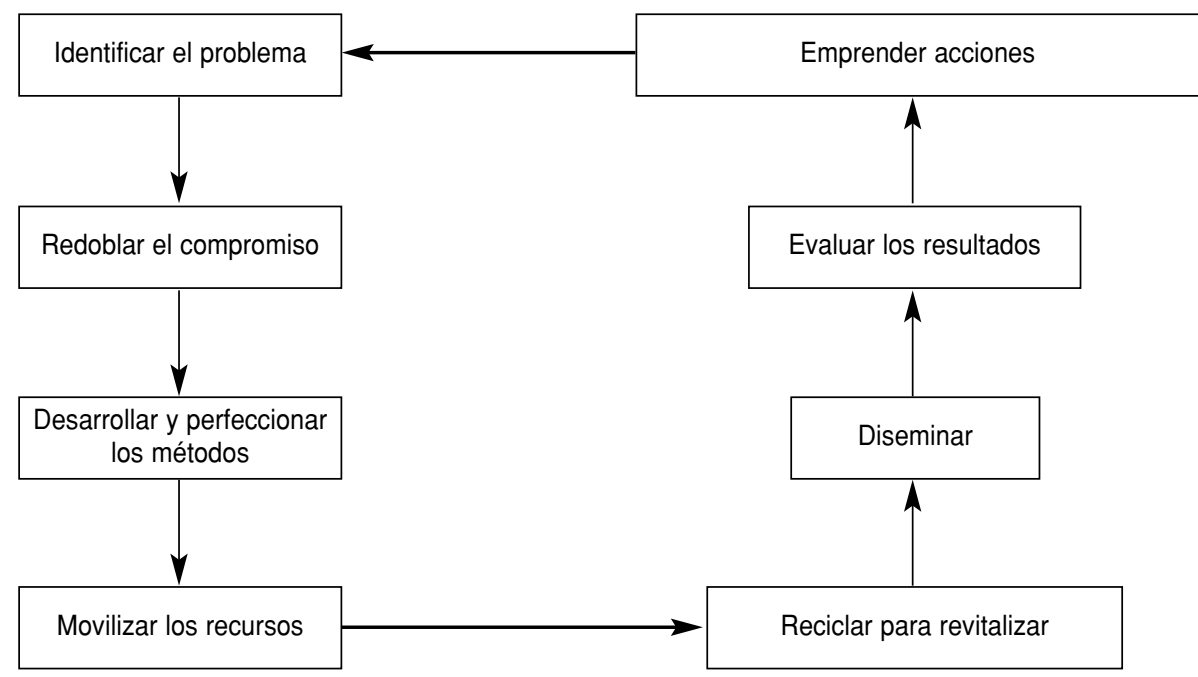

rán a los planificadores a evitar ciertos errores, como el de emprender acciones sin disponer de los recursos necesarios para completarlas. El modelo a base de "preceder" y "proceder", aplicado con gran frecuencia en el campo de la promoción de la salud (Green y Kreuter, 1991), ofrece directrices similares. Aunque estos pasos representan una progresión que se produce con naturalidad dentro de un proyecto de salud, la secuencia raras veces se reconoce en sentido formal. Sin embargo, versiones análogas de este modelo se aplican con frecuencia en las escuelas comerciales.

Primer paso: identificar el problema. En una campaña local o nacional, este paso se produce cuando unas cuantas personas con iniciativa, alarmadas por la apreciación de un problema de salud cardiovascular, reúnen información por medio de encuestas, sondeos y evaluaciones de las necesidades de la comunidad con el propósito de establecer metas y elegir posibles áreas de intervención. En cada campaña el tema ha de ser específico y los organizadores deberán elegir entre un enfoque amplio en que se combaten simultáneamente varios factores de riesgo —entre otros, falta de ejercicio, alimentación poco nutritiva, hipertensión, sobrepeso, tabaquismo, abuso de alcohol y estrés-, o concentrar sus esfuerzos en una sola área.

El proceso de identificación debe basarse en encuestas que recojan información de salud de carácter nacional o local. Esta información debe poner de manifiesto la magnitud del problema y su importancia en relación con otros que también exigen un consumo de recursos.

Segundo paso: redoblar el compromiso. En un entorno comunitario, los iniciadores de un programa frecuentemente necesitan ampliar sus bases mediante un proceso de reclutamiento, organización y entrega de poder a individuos e instituciones dentro de su comunidad. Las alianzas llevarán a la formación de agrupaciones de individuos y organizaciones (profesionales de la salud, agencias de salud voluntarias, lugares de trabajo, grupos de ciudadanos, unidades de salud pública, políticos y demás). Este proceso es fundamental para poder crear condiciones que permitan recibir y ampliar los programas educativos y normativos instituidos.
Como ilustran muchos de los ejemplos descritos en la Declaración de Cataluña, en ocasiones hace falta un período de incubación de varios años para que un grupo de personas relativamente pequeño cobre fuerza hasta el punto de poder aplicar programas completos, especialmente cuando existe la oposición de intereses políticos o comerciales. Como se observara en el caso de las iniciativas para la gravación del tabaco en Victoria y California (capítulo 3), los defensores de la salud tuvieron que esforzarse mucho para conseguir el apoyo público que permitió modificar las leyes en vigencia. En la práctica, una sola institución suele tomar la iniciativa dentro de una coalición, pero se necesita un gran esfuerzo colectivo para vencer el proteccionismo y lograr que los recursos se compartan.

Tercer paso: desarrollar y perfeccionar los métodos. Una vez que se ha establecido la necesidad de crear un programa y que se han formado coaliciones o grupos de trabajo, el equipo deberá destinar el tiempo necesario para planificar acciones específicas. Estas deben adecuarse a la tarea y también a las necesidades, preferencias, recursos y capacidad de las personas a quienes está destinado el programa.

$\mathrm{Si}$ se desea investigar las verdaderas necesidades de la población destinataria, a veces este paso debe incluir investigaciones formativas (por sondeos y encuestas) o el desarrollo de proyectos piloto.

El grupo o los grupos formados con el fin de ejecutar un plan de acción pueden utilizar esta información para llegar a un consenso sobre la idoneidad de las metodologías e instrumentos disponibles.

Cuarto paso: movilizar los recursos. Como es lógico, este paso preliminar es necesario para poder dar un fuerte impulso a los programas establecidos. Cuando no hay recursos, en lugar de abandonar el proyecto los organizadores a veces pueden adaptar sus planes a proyectos de menor escala. Los pro- 
yectos pueden convertirse en programas piloto para comprobar la eficacia de los métodos elegidos o convencer a los demás de que el proyecto merece un mayor aporte de recursos.

Quinto paso: emprender acciones. Este paso puede abarcar acciones de muy diversa índole, desde las más importantes hasta las más pequeñas, pero debe incluir los siguientes elementos:

- educación;

- cambios ambientales;

- cambios normativos.

La educación. En general, los programas que dan buenos resultados se valen de diversos mecanismos para transmitir mensajes educativos de refuerzo con regularidad. Constituyen ejemplos la radio y la televisión, las festividades en la comunidad, las clases, talleres, periódicos y boletines, la amplia distribución de materiales impresos de autoayuda, los videos y sistemas educativos computadorizados y los voluntarios que instruyen a la población mediante la transmisión verbal de sus mensajes de un habitante a otro.

La secuencia de medidas educativas se ha de basar en principios teóricos sólidos que orienten al público durante el proceso de definir un plan de acción, mejorar sus conocimientos, adquirir las habilidades requeridas y conservar los hábitos aprendidos (Farquhar, et al., 1991). El ambiente social abarca componentes de los ambientes físico e informativo, pero incluye un análisis más extenso de algunos problemas, como serían el empleo y los servicios sociales y educativos, en que también se necesitan cambios para poder lograr un estado óptimo de salud cardiovascular.

Cambios ambientales. Puede referirse a cambios en el ambiente físico, como la creación de áreas libres de humo y de mejores instalaciones para hacer ejercicio, así como a cambios en el ambiente informativo. Este último, que frecuentemente se pasa por alto, comprende la publicidad, los espectáculos, los medios de comunicación, los rótulos que llevan los alimentos y otras fuentes de información.

Cambios normativos. El tercero de los procesos interactivos que corresponden al área de implementación se asocia con cambios en las leyes y reglamentos. Este componente es un aspecto importante de las intervenciones que se inician en los niveles superiores del gobierno. Por ejemplo, un cambio en el impuesto sobre los cigarrillos puede tener un efecto notable sobre la prevalencia del tabaquismo. Entre los cambios iniciados en los niveles superiores también puede haber regulaciones nuevas en torno a distintos asuntos, como el reembolso por las compañías de seguros - orientados, por ejemplo, a dar a los médicos un incentivo para el ejercicio de la medicina preventiva- o nuevas normas para la diseminación de información por los medios publicitarios. Los cambios normativos también pueden resultar muy efectivos en el nivel del gobierno local. Un ejemplo está dado por las leyes que prohíben fumar en restaurantes o lugares de trabajo, con su poderoso efecto disuasor.

Sexto paso: evaluar los resultados. El proceso de evaluación es parte fundamental de cualquier programa, no solo para verificar si se alcanzaron o no los objetivos, sino también para proporcionar indicadores que marquen el rumbo futuro. La evaluación del proceso de cambio (es decir, la determinación de la manera en que los cambios se obtuvieron) puede resultar tan importante como la evaluación de los resultados de un programa o intervención.

Séptimo paso: difundir los resultados. La demostración de buenos resultados durante la fase de evaluación naturalmente facilitará la tarea de alentar a otros a adoptar el programa.

Muchos opinan que la difusión ocurre automáticamente a partir del momento en que la población se entera de los buenos resultados. Sin embargo, la diseminación exige gran esfuerzo y atención. Por ejemplo, resultará mucho más eficiente $\mathrm{y}$, por ende, efectivo en función del costo, convencer al ministerio de educación de una provincia de que adopte en todo el sistema un plan de estudios sobre la salud cardiovascular, que intentar persuadir individualmente a docenas o centenares de escuelas. También es importante cerciorarse de que los programas se apliquen de una manera que garantice su efectividad en el largo plazo.

Uno de los propósitos de la Declaración de Cataluña es estimular la difusión y también las investigaciones en este campo, que tanta falta hacen (Farquhar, 1996). Hay motivos, sin embargo, para abrigar optimismo, como revelan los numerosos triunfos aquí descritos. Se espera que estas intervenciones productivas lleven a muchos miembros de la comunidad internacional a adoptar, adaptar y diseminar algunos de estos éxitos notables.

Octavo paso: reciclar. A veces es preciso reciclar, volviendo al primer paso, si no se obtuvieron resultados suficientemente buenos o si las condiciones han cambiado de tal modo que se torna necesario idear nuevas estrategias.

\section{CAPÍTULO 6}

\section{INVERSIONES FRUCTÍFERAS EN LA SALUD CARDIOVASCULAR}

La Declaración de Cataluña cierra con una serie de ejemplos que ilustran los beneficios de invertir en la salud cardiovascular. En cierto sentido, todos los relatos destacados en este documento constituyen ejemplos de intervenciones fructíferas en la salud cardiovascular. En algunos casos, especialmente cuando se trata de programas ofrecidos en clínicas, sitios de trabajo o en el seno de la comunidad, los beneficios económicos superan a los costos; en otros, sin embargo, los beneficios económicos son menos patentes. 
A menudo, el éxito de la inversión se puede apreciar cuando se adopta un punto de vista más amplio. Por ejemplo, una inversión en la salud cardiovascular puede ser fructífera cuando los diferentes miembros de una alianza aúnan sus recursos. Otro ejemplo es el fortalecimiento del sector social, especialmente de aquellos componentes relacionados con la salud pública y la atención de la salud.

El buen rendimiento de una inversión se mide no solo en términos de proyectos y programas que producen ahorros directamente mensurables, sino también de los que generan modificaciones favorables de los factores de riesgo de enfermedad cardiovascular, especialmente cuando se observan en el largo plazo. Los programas y actividades que se realizan en un contexto clínico o laboral naturalmente ofrecen mejores oportunidades de medir tanto los costos como los beneficios. La presente sección comienza con estas dos categorías.

\section{La inversión en la salud cardiovascular en el medio laboral}

Ya se han mencionado inversiones rentables en la salud cardiovascular dentro del entorno laboral. En el capítulo 3 se describió un programa de este tipo en Electrostal, Rusia. El programa multifactorial de promoción de la salud que se introdujo en esa instalación en los años ochenta se dirigió en gran medida a combatir las enfermedades cardiovasculares y claramente representó una inversión rentable en la salud cardiovascular. En un plazo relativamente corto se observó una notable disminución de la morbilidad y mortalidad por estas enfermedades, junto con una reducción del absentismo laboral de 30\%. Estos resultados han llevado a la propia empresa a continuar el programa.

En el capítulo 3 también se cita el ejemplo de cuatro fábricas pertenecientes a la gran compañía estadounidense General Motors, fabricante de automóviles cuya sede se encuentra en el estado de Michigan. Como ocurrió en Electrostal y como suele suceder en casi todos los programas ofrecidos en el medio laboral, las intervenciones realizadas en esta fábrica automotriz emanaron del nivel superior. Hubo, sin embargo, distintos grados de activismo entre los propios trabajadores, de manera que un movimiento ascendente simultáneo se originó entre el personal médico y de salud ocupacional de la compañía. En este estudio, cuyo diseño fue experimental, las dos plantas donde se efectuaron las intervenciones más intensas produjeron el mayor rendimiento. En ellas la razón de beneficio a costo fue cinco a seis veces mayor que en las otras plantas (Erfurt, et al., 1991).

De los programas ofrecidos en Rusia y los Estados Unidos se desprende una lección sobre cómo hacer una inversión rentable: los programas contra el conjunto de los principales factores de riesgo de enfermedad cardiovascular en que se aplican varias estrategias (como el tamizaje, la educación, los materiales de autoayuda y la participación familiar) prometen ser los más efectivos en función del costo. Se llegó a conclusiones similares tras una extensa revisión de 23 programas bien diseñados de promoción de la salud instituidos en los lugares de trabajo, cuyo rendimiento fue de US\$3,00 por cada US\$ 1,00 invertido en los programas, razón que se asemeja a las aquí descritas (Pelletier, 1993). Como se mencionara anteriormente, la inversión en la salud cardiovascular se justifica plenamente; basta con advertir la frecuencia declinante (o ascendente) de los trastornos cardiovasculares en los países. Pero incluso en un sentido más circunscrito, la inversión también puede justificarse sobre la base exclusiva de los resultados de programas ofrecidos en el entorno laboral, ya que en ellos se ejerce un mayor control sobre la medición de costos y beneficios.

\section{La atención primaria y los programas en entornos clínicos}

En los entornos donde se brinda atención clínica, al igual que en los lugares de trabajo, la medición de resultados suele ser mejor que en los programas efectuados en unidades más extensas, como la comunidad, la provincia, o el país. Un estudio en Israel (recuadro 36) no solo produjo beneficios apreciables en función del costo, sino que también demostró el valor de conseguir una buena colaboración inicial entre centros clínicos en puntos geográficos distintos.

En el capítulo 3 también se describió un programa en Houston, Texas, que demostró que varias clínicas pertene-

\section{RECUADRO 36. El tratamiento no farmacológico de la hipertensión arterial en Israel}

Un estudio llevado a cabo en Israel para determinar la efectividad del tratamiento no farmacológico de la hipertensión arterial demostró que un equipo bien adiestrado compuesto de un médico y un miembro del personal de enfermería dedicados a dar instrucción individual a un paciente puede controlar muchos de los factores de riesgo de enfermedades cardiovasculares susceptibles de modificación y al mismo tiempo reducir a un mínimo la medicación. Los pacientes que participaron en el estudio, que fueron tratados en cinco clínicas de atención primaria dispersas por todo el país, redujeron el gasto en medicamentos en $44 \%$ como resultado de la intervención.

Este estudio ha sido extendido a otras siete unidades de atención primaria, donde también ha producido buenos resultados. Al cabo de 6 meses, los pacientes estaban gastando $20 \%$ menos en medicamentos. Actualmente los médicos y miembros del personal de enfermería dedicados a la atención primaria están recibiendo adiestramiento sobre la administración de intervenciones no farmacológicas intensivas para el tratamiento de la hipertensión y de otros factores de riesgo de las enfermedades cardiovasculares. Este proyecto tiene el apoyo financiero de la CINDI en Israel, así como del Ministerio de Salud y de la Asociación Israelí de Medicina Familiar.

Fuente: J. Viskoper, comunicación personal, 1995. 
cientes a una red de centros de atención primaria lograron aumentar el porcentaje de pacientes con hipertensión arterial controlada de 33 a 79\% (Vallbona, et al., 1985). Este programa ilustra la capacidad de una escuela de medicina para mejorar la atención primaria en una extensa zona metropolitana.

En Moscú, el Programa Multifactorial para la Prevención de las Enfermedades Cardiovasculares (Chazova, et al., 1989) alcanzó a 3000 personas por medio del sistema de centros clínicos existente. En un período de 5 años este programa redujo la mortalidad por enfermedades cardiovasculares a una tasa $41 \%$ más baja que la de un grupo control de 3000 personas. Este efecto tan marcado confirma los beneficios de un programa multifactorial llevado a cabo en los centros de atención clínica.

En California se obtuvieron resultados de similar magnitud en el ámbito de la prevención secundaria mediante un estudio aleatorio de 600 sobrevivientes con antecedentes de un primer episodio de enfermedad coronaria (descrito en el capítulo 3). En un período de 4 años, el Proyecto Stanford de Intervención contra el Riesgo de Cardiopatía Coronaria redujo el número de recidivas de infarto del miocardio y de reintervención quirúrgica coronaria en el grupo sometido a la intervención a la mitad del observado en un grupo control tratado por un cardiólogo con métodos convencionales. Como se señalara anteriormente, la razón de beneficio a costo al compararse el grupo sometido a la intervención y el grupo control fue de 2:1 para todos los 4 años y de 4:1 para los últimos tres.

Un último ejemplo de un programa para la prevención de las enfermedades cardiovasculares ubicado en un entorno clínico es el del Hospital de Lehigh Valley en Allentown, Pensilvania, Estados Unidos (Nester y Thieme, 1995). Este ejemplo es típico de una tendencia promisoria que se observa en hospitales estadounidenses, muchos de los cuales actualmente buscan establecer contacto con la comunidad por medio de programas preventivos, sin dejar de cumplir con su función tradicional de atender al enfermo. El hospital de Lehigh Valley, en colaboración con médicos locales, empezó a ofrecer programas globales para combatir los factores de riesgo de las enfermedades cardiovasculares. También se vinculó con otros hospitales, al principio siete y posteriormente algunos más, para cubrir una zona más extensa. Su vinculación con otros hospitales locales le permite cubrir un territorio más extenso. No solo lograron ofrecer buenos servicios de atención primaria (mediante la formación de alianzas descrita en el capítulo 3), sino que lo hicieron aprovechando los recursos disponibles en las comunidades servidas. De esa manera lograron demostrar cómo las instituciones locales pueden reestructurar sus prioridades y lograr buenos resultados sin necesitar fondos adicionales.

En el capítulo 4 se examinó cómo la aversión al cambio constituye uno de los obstáculos que impiden el mejoramiento de la salud cardiovascular. Los programas ofrecidos en contextos ocupacionales y clínicos que se describen en este documento ilustran claramente que la aversión al cambio se puede superar si se demuestra que los programas destinados a mejorar la salud pueden ser efectivos sin ser caros. Estos programas son aun más alentadores: demuestran cómo las instituciones y los profesionales lograron cambios importantes modificando las opiniones, costumbres e incluso los hábitos sanitarios más arraigados de los usuarios, es decir, de las poblaciones destinatarias y de los proveedores de atención de salud y formuladores de políticas. Sin embargo, la principal lección que nos enseñan los ejemplos tomados del entorno clínico es que la falta de colaboración entre profesionales de la salud y los formuladores de políticas (capítulo 4) se puede superar.

\section{Programas comunitarios de carácter global}

La China es un país con una larga historia de programas de educación para la salud que han dado buenos resultados. En las últimas décadas, casi todos los programas se han destinado a la lucha contra las enfermedades transmisibles, pero recientemente las autoridades de salud del país han empezado a preocuparse por el rápido incremento de las enfermedades cardiovasculares y por la fuerte posibilidad de que en la próxima década estas lleguen a convertirse en la principal causa de mortalidad de la población. Por consiguiente, en muchas partes de la China se ha hecho un esfuerzo muy grande por reorientar los métodos conocidos de educación en salud hacia la prevención de los trastornos cardiovasculares. Entre ellos se puede citar el Programa de Intervención contra las Enfermedades Cardiovasculares de Tianjin (recuadro 37). Esta iniciativa bien organizada e innovadora para la promoción de la salud ofrece un excelente ejemplo de que un país en transición no necesariamente tiene que reaccionar demasiado tarde cuando una nueva epidemia asoma en el horizonte.
RECUADRO 37. Tianjin, China: Programa de Intervención contra las Enfermedades Cardiovasculares

En 1991, la ciudad de Tianjin, China, inició un programa comunitario contra las enfermedades cardiovasculares. Se emplearon concursos televisados, cuestionarios en la prensa, incentivos económicos, consultas domiciliarias y la distribución de recetas de alimentos con bajo contenido de sodio, a fin de dirigir a 400000 personas programas de intervención para el control de la hipertensión arterial, el tabaquismo y el consumo de sal. Desde la iniciación del programa se han observado cambios de conducta, entre ellos la creación de muchos ambientes nuevos libres de humo y el aumento de las ventas de alimentos bajos en sodio.

Aprovechando el éxito del programa de Tianjin, el Banco Mundial está financiando en siete ciudades chinas programas para la prevención de las enfermedades crónicas, dirigidos principalmente a combatir los principales factores de riesgo cardiovascular: tabaquismo, alimentación poco nutritiva, sedentarismo y descontrol de la tensión arterial.

Fuentes: J. Kaplan, comunicación personal, 1991. 
Otro estudio en los Países Bajos constituyó una iniciativa de colaboración entre un centro de salud comunitario y el Departamento de Medicina Social de la universidad estatal. El estudio abarcó todos los factores de riesgo conductuales que se asocian con las enfermedades cardiovasculares y tuvo una amplia participación comunitaria. Las organizaciones locales participaron en intervenciones mixtas en que se incluyeron los medios de comunicación, actividades en grupo y actividades de carácter social (de Vries, et al., 1995). Iniciado en 1991, este proyecto ha logrado una mayor concienciación pública, la formación de grupos para llevar a cabo proyectos intersectoriales y la reducción del tabaquismo. Se está planificando su diseminación, conducente a la creación de un programa comunitario para toda la región. El proyecto muestra cómo vencer la barrera de las actitudes convencionales y el uso extenso de personal voluntario comprueba que un proyecto manejado con imaginación puede sacar un máximo rendimiento a los recursos disponibles.

La ciudad de Bremen, Alemania, también ha notificado el éxito de una campaña innovadora para la reducción de varios factores de riesgo cardiovascular (recuadro 38). Este estudio fue financiado por el gobierno alemán y llevado a la práctica por la Universidad de Bremen con la plena participación de muchas organizaciones locales.

Dos de los programas de intervención más conocidos de carácter general y comunitario (los de Stanford y Carelia del Norte, capítulo 3) ofrecen ejemplos de alta efectividad y abundantes beneficios en función del costo. El Estudio Stanford en Tres Comunidades (1972-1975) produjo resultados sorprendentemente buenos mediante el uso de la radio, la televisión y los periódicos y la amplia circulación postal de materiales de autoayuda, pese a que no se aplicaron técnicas de promoción de la salud más agresivas (y caras), como clases o sesiones de asesoramiento. Este proyecto demostró la efectividad del enfoque propagandístico, que es inherentemente efectivo en función del costo (Farquhar, et al., 1977).

RECUADRO 38. Estudio Alemán sobre la Prevención de las Enfermedades

Cardiovasculares en la ciudad de Bremen

Entre 1985 y 1991 la ciudad de Bremen, puerto situado en el norte de Alemania, estuvo incluida en el Estudio Alemán sobre la Prevención de las Enfermedades Cardiovasculares. Se trata de un proyecto comunitario de demostración para la prevención de los ataques al corazón y accidentes cerebrovasculares cuyo propósito es modificar los factores de riesgo de estas enfermedades en la población. En Bremen, las iniciativas se dirigieron principalmente a dos distritos habitados en su mayor parte por obreros (200 000 habitantes). Para llevar a cabo campañas preventivas se creó una red comunitaria con participación de los medios de comunicación (televisión, radio y prensa locales), profesionales de la salud pública (médicos, odontólogos y farmacéuticos), fundaciones de ayuda para enfermos, asociaciones deportivas y asociaciones profesionales de gerentes de cafeterías en entornos laborales y de cocineros de restaurantes.

La mayor parte de las actividades fueron organizadas o coordinadas por el personal del Estudio Alemán sobre la Prevención de las Enfermedades Cardiovasculares y en puntos de encuentro a la entrada de almacenes para tratar cuestiones de salud en los distintos distritos. Se formaron coaliciones provisionales con los gremios de reposteros y supermercados. Los políticos estatales y locales, así como actores y atletas y otros miembros clave de la comunidad, fueron incluidos en los espectáculos públicos.

Al final del estudio se logró modificar algunos factores de riesgo mediante el control de la hipertensión y del tabaquismo (en hombres solamente) y la modificación de los hábitos nutricionales (de las mujeres especialmente y de todas las personas con concentraciones altas de colesterol). Cuando se comparó con el resto de Alemania, Bremen tuvo una tasa mucho más baja de factores de riesgo después de la intervención. Los efectos sobre el control de la hipertensión pudieron vincularse con el empeño de los médicos en esa área, mientras que los cambios en otros factores de riesgo se atribuyeron a la mezcla de intervenciones propias del programa. Después de terminado el estudio en 1991, varias organizaciones en otras partes de Alemania, entre ellas las ciudades de Bremerhaven, Dresden y Rostock, adoptaron módulos propagandísticos exitosos. Actualmente se están aplicando en toda Alemania medios especiales para llevar a cabo las intervenciones. Entre ellos figuran las obras Heart Healthy Cookbook y Kindergarten Cookbook.

Fuente: E. Greiser, 1992.

El Proyecto Stanford en Cinco Ciudades (1978-1996), que fue más grande, también tuvo un gran efecto sobre el riesgo de enfermedad cardiovascular, pese a la cantidad relativamente pequeña que se gastó en la campaña: alrededor de US $\$ 2,00$ per cápita, procedentes de fuentes ajenas a la comunidad. Ello equivale a cerca de $1 \%$ del costo de los cigarrillos adquiridos por los 22000 fumadores en una población total de 135000 habitantes. La plena colaboración de las escuelas, hospitales, agencias de salud voluntarias y profesionales de la salud contribuyó a la efectividad del programa en relación con el costo. Estas alianzas representan un cambio en la forma de proceder establecida, sin que ello implique gastos adicionales para los colaboradores. Un indicador de éxito financiero de igual importancia es el hecho de que el departamento de salud del condado, que colaboró en la etapa inicial, haya aumentado por un factor de nueve al personal dedicado a la promoción de la salud. Actualmente, gracias a la ayuda de los hospitales, profesionales de la salud y políticos locales, el departamento de salud aplica a otros problemas sanitarios los métodos de promoción de la salud aprendidos durante su trabajo con la Universidad de Stanford, mientras continúa batallando contra las enfermedades cardiovasculares. Esta adopción por una comunidad de métodos desarrollados colectivamente y la continuación de los programas mismos representan la meta final de cualquier proyecto de demostración comunitario.

El famoso proyecto de Carelia del Norte, que se inició en 1972, también provee un ejemplo patente de una inversión muy rentable. Un resultado notable ha sido que el gran descenso de las enfermedades cardiovasculares producido por el programa redundó en grandes ahorros en pensiones médi- 
cas que de lo contrario se hubieran destinado a individuos con trastornos cardiovasculares. Estos ahorros compensaron, con creces, el costo del programa de intervención (Puska, et al., 1983). El proyecto, que se llevó a cabo en todo el condado por un período de dos décadas, también ha producido una reducción de ataques al corazón y accidentes cerebrovasculares significativamente mayor que la observada en el condado adyacente y en el resto de Finlandia. De ahí que en términos monetarios y humanos, los beneficios hayan superado a los costos. Como sucedió en los proyectos de Stanford, Carelia del Norte movilizó coaliciones y creó alianzas productivas con las instituciones de salud, médicos, clubes de mujeres, agencias voluntarias, medios de comunicación y escuelas locales.

\section{El sector voluntario}

Las fundaciones de salud cardiovascular de distintos países han desempeñado un papel importante en la prevención de las enfermedades cardiovasculares (capítulo 3). Si se tiene en cuenta el gran número de voluntarios que utilizan, su función en la formación de alianzas y sus logros en el campo de la educación pública, la inversión que han hecho estas fundaciones puede declararse un éxito. Otros grupos del sector voluntario dedicados a combatir el cáncer, las enfermedades pulmonares y el tabaquismo también han dado buenos resultados. Como ilustrara el ejemplo presentado en el capítulo 3, estas organizaciones contribuyeron mucho a la adopción del impuesto sobre el tabaco en California. Tanto en Europa como en las Américas se han dado ejemplos de una coordinación regional productiva entre las fundaciones que combaten los trastornos cardíacos y cerebrovasculares.

\section{El sector privado}

A continuación se describen dos programas comunitarios que han rendido beneficios en el campo de la nutrición y que han tenido la partici- pación del sector privado. Como parte de una campaña nacional denominada "Cuidado con las grasas", la Fundación Neerlandesa del Corazón ha organizado visitas a mercados dirigidas por nutricionistas para grupos de adultos. La finalidad específica de las visitas ha sido estimular a los participantes a reducir el contenido graso de los comestibles que compran y, según los resultados, $75 \%$ de los adultos y $63 \%$ de los niños en edad escolar redujeron su consumo de grasa. Los administradores reaccionaron con entusiasmo porque se estrecharon las relaciones con los consumidores. La actividad, que recibió gran atención en todos los medios de difusión, se está extendiendo a otras comunidades (van Dis, et al., 1995).

Un ejemplo adicional de colaboración con la industria de alimentos del sector privado fue notificado por un distrito minero en Nueva Gales del Sur, donde la tasa de mortalidad por enfermedades cardiovasculares es la más alta de la costa oriental de Australia. En ese territorio se solicitó a varios establecimientos que servían comida rápida que modificaran la preparación de los alimentos fritos inmersos en aceite, procurando reducir la grasa absorbida. Los establecimientos también cambiaron el tipo de grasa, usando aceites con una elevada pro- porción de ácidos grasos monoinsaturados en lugar de las mantecas sólidas usadas habitualmente. El consumo de grasas saturadas se redujo de $6 \mathrm{~g}$ a menos de $1 \mathrm{~g}$ por cada $100 \mathrm{~g}$ de patatas fritas, sin que los consumidores advirtieran ningún cambio en el sabor. Esta intervención relativamente sencilla debe alentar a las personas que ya no creen en la industria de alimentos rápidos y reforzar su fe en la colaboración de la industria alimentaria para obtener beneficios de salud (Roberts, et al., 1995).

\section{Programas nacionales}

Existen muy pocos programas nacionales y uno de ellos es "El gran plan de ejercicio filipino" (recuadro 39). El programa, que fue iniciado por el Ministerio de Salud de las Filipinas al constatarse que $25 \%$ de las defunciones en el país se debían a enfermedades cardiovasculares, demuestra claramente que la identificación de un "nuevo" problema de salud puede dar ímpetu a la definición de una política nacional. Ha demostrado, además, un hecho más importante: que un país en desarrollo puede poner en marcha programas de prevención a gran escala antes de que la epidemia emergente de enfermedades cardiovascula-

RECUADRO 39. El gran plan de ejercicio filipino

Las Filipinas, país integrado por 7000 islas, ha conseguido movilizar los recursos de todos los sectores en su territorio nacional mediante una campaña de gran originalidad efectuada por todos los medios propagandísticos. La Declaración de Cataluña hace un llamado mundial para cerrar la brecha representada por las desigualdades en el campo de la salud. Con miras a prevenir una epidemia, las Filipinas, país en desarrollo, actualmente destina sus recursos a combatir las desigualdades que existen en su territorio.

El Centro Cardiovascular de las Filipinas ha notificado que en 1988-1989 40\% de los adultos fumaban, $24 \%$ tenían hipercolesterolemia y $50 \%$ llevaban una vida sedentaria. Gracias a la colaboración de muchos sectores, en 1994 un Comité Directivo Nacional y un Grupo Técnico de Trabajo iniciaron un programa nacional llamado "El gran plan de ejercicio filipino".

Este proyecto tiene la participación de todos los sectores de la sociedad, entre ellos el de las organizaciones gubernamentales y no gubernamentales y el sector privado. Todos los grupos de actividad física han sido movilizados en un intento por diseñar un programa de ejercicio de 15 a 20 minutos que sea propiamente filipino y que mejore el estado de salud cardiovascular. Con el proyecto se espera lograr que todos los habitantes de las Filipinas participen en "El gran plan de ejercicio filipino", independientemente del lugar donde se encuentren: en la comunidad, en las escuelas o en instalaciones industriales.

Fuente: M. Narvaez, 1995. 
res alcance la magnitud que despliega en los países desarrollados.

Como ya se ha comentado en este informe (capítulo 3), otros países también han logrado resultados similares en todo su territorio: la Comisión Nacional Guatemalteca contra el Tabaquismo, el éxito de Portugal en su lucha contra la hipertensión y los planes nacionales de Túnez para la prevención de las enfermedades cardiovasculares en el sector de la atención primaria. Alemania (como ya se señaló en este capítulo, recuadro 38) y la República Checa (recuadro 40) recientemente crearon programas nacionales que refuerzan notablemente el compromiso de luchar contra las enfermedades cardiovasculares.

\section{Programas internacionales y regionales}

Como se explicó en el capítulo 3, el Comité Latinoamericano Coordinador del Control del Tabaquismo (CLACCTA) es un buen ejemplo de una organización de alcance regional que, desde su fundación en 1993, se ha ganado el apoyo de numerosos países latinoamericanos. La iniciativa antitabáquica de Guatemala (descrita en el capítulo 3) es uno de los programas auspiciados por el CLACCTA. Resulta evidente que un programa regional de este tipo representa un triunfo en términos de colaboración y formación de alianzas. En términos de inversión, sus buenos resultados obedecen en parte a la combinación de recursos que representa y al uso compartido de información y recursos que tuvo lugar. Hay grandes posibilidades de que aporte futuros beneficios para la salud.

La Red China Internacional para la Salud Cardiovascular (descrita en el capítulo 3) tiene pocos años de existencia, pero ya es una inversión rentable: no solo ha contribuido al intercambio de tecnologías, sino que representa una singular unión de las comunidades chinas del litoral pacífico y encierra la esperanza de que en el futuro haya mayores posibilidades de prevenir las enfermedades.

El programa InterHealth y la Intervención Integrada de Alcance Nacional contra las Enfermedades No Transmisibles (Countrywide Integrated Noncommunicable Disease Intervention, CINDI), descritos en el capítulo 3, también son ejemplos notables de colaboración internacional. En algunos casos el ahorro en costos es un aspecto inherente, gracias a la eficiencia con que se comparten las tecnologías aplicadas en las intervenciones. La región del Pací- fico occidental ha obtenido muchos buenos resultados en los últimos años en cuanto al establecimiento de políticas antitabáquicas, generados en parte por iniciativa de una persona dedicada a esa causa: Judith Mackay de Hong Kong (recuadro 41).

RECUADRO 41. Políticas

antitabáquicas en el Pacífico occidental

En años recientes, la región del Pacífico asiático se ha convertido en el blanco principal de las empresas tabacaleras transnacionales. En el número de septiembre de 1986 de la revista industrial World Tobacco, un relato sobre "El gran futuro proyectado para el Pacífico occidental" examinó el "crecimiento potencial" del comercio en ese territorio y el deseo de aumentar el número de fumadores. La industria ha pronosticado que entre 1991 y 2000 las ventas de tabaco en la región se incrementarán en 33\%.

A pesar del empeño de la industria tabacalera, los países del Pacífico asiático han tomado diversas decisiones políticas en las últimas dos décadas para limitar el uso del tabaco. Los siguientes ejemplos ponen de manifiesto los logros obtenidos en toda la región (algunos con el apoyo del Grupo Consultivo Asiático sobre el Tabaco, con sede en Hong Kong) y respaldan la noción de que las políticas deben desempeñar un papel fundamental en la limitación del consumo de productos del tabaco y, por consiguiente, en la reducción de la incidencia de las enfermedades cardiovasculares.

En 1971, Singapur prohibió la comercialización de los productos del tabaco.

En 1983 la China se convirtió en el primer país en prohibir que se fume en todos los vuelos internos.

En 1987, Hong Kong prohibió la fabricación, importación y venta de productos a base de tabaco libre de humo.

En 1993, las Filipinas lanzaron una campaña representada por la mascota nacional Yosi Kadiri, con objeto de concienciar al público sobre los peligros del tabaquismo.

En 1994, Shanghai impuso reglamentos gubernamentales que prohíben fumar en prácticamente todas las áreas públicas en el interior de los edificios;

Tailandia ha llevado a cabo una campaña nacional encaminada a contrarrestar la presión comercial de las tabacaleras estadounidenses y a mantener vigente la prohibición nacional contra su propaganda.

Fuente: J. Mackay, comunicación personal. 


\section{CONCLUSIONES}

En la Declaración de Cataluña se subraya el concepto de que la inversión en la salud cardiovascular es esencial, tanto por sus consecuencias humanitarias como por los beneficios económicos que conlleva. Se proporcionan ejemplos prácticos, principalmente en forma de relatos, para describir el desarrollo de un programa en todas sus etapas. Citamos las palabras de Margaret Mead: "Jamás hay que dudar de que unos cuantos individuos tesoneros pueden cambiar el mundo. De hecho, ha sido siempre la única manera en que las cosas han cambiado". Esperamos que la Declaración de Cataluña ayude al lector a sentirse más dedicado que nunca al postulado de la Declaración de Victoria que la precedió, según el cual ya contamos con los conocimientos y métodos necesarios para prácticamente eliminar los ataques al corazón y los accidentes cerebrovasculares de la faz de la tierra. Si el lector ha redoblado su dedicación a esta meta, la Declaración habrá cumplido su cometido.

\section{RECOMENDACIONES}

\section{Todo individuo y grupo interesado en la salud cardiovascular}

1. Toda entidad interesada en apoyar las actividades para la promoción de la salud cardiovascular debe percatarse de la versatilidad y posibilidad de cambio que poseen las comunidades cuando están bien informadas, organizadas $\mathrm{y}$ movilizadas, y debe establecer alianzas con ellas.

\section{El sector de la salud y el sector privado}

2. Los organismos de salud internacionales, los departamentos de salud, las agencias de atención voluntarias, las organizaciones de profesionales de la salud, los educadores, la comunidad académica y el sector privado deben emprender las siguientes medidas:

a) apoyar el desarrollo de programas comunitarios para el fomento de la salud cardiovascular mediante el aprovechamiento de los recursos apropiados del sector de la salud y de otros sectores;

b) promover actividades en pro de la salud cardiovascular, tanto en países desarrollados como en desarrollo, encaminadas a evitar que los niños y jóvenes adquieran hábitos malsanos;

c) formar alianzas para la defensa de la salud cardiovascular y la ejecución de políticas globales para su promoción y la prevención de las enfermedades del corazón, en consonancia con los lineamientos de la Declaración de Victoria;

d. desarrollar estrategias y asignar los recursos adecuados para aplicar los conocimientos y experiencias obtenidos mediante intervenciones en pro de la salud cardiovascular, lo cual incluye movilizar a la comunidad y crear programas en entornos laborales y escolares para prevenir dichas enfermedades y promover la buena salud;

e) proporcionar los recursos para crear y mantener alianzas que apoyen el desarrollo de políticas y programas en pro de la salud cardiovascular, intercambiar conocimientos y experiencias, y ayudarse mutuamente a identificar fuentes de financiamiento que aseguren la coordinación y el sostenimiento de las actividades.

\section{Las organizaciones de salud voluntarias}

3. Las organizaciones de salud voluntarias, especialmente las asociaciones que luchan contra las enfermedades cardiovasculares, el cáncer, los trastornos pulmonares, el tabaquismo y la diabetes, deberán proporcionar asistencia técnica y los recursos apropiados a los países y regiones del mundo que deseen establecer organizaciones de asistencia voluntaria con estructuras similares.

\section{Los servicios de salud}

4. Los departamentos de salud y otras agencias sanitarias, en asociación con las correspondientes agencias, organizaciones y coaliciones comunitarias del sector de la salud y de otros sectores, deberán emprender las siguientes acciones:

a) Iniciar programas de salud cardiovascular utilizando, siempre que sea posible, el sistema de salud existente (por ejemplo, salud pública y atención primaria);

b) apoyar actividades que mejoren la efectividad de las prácticas preventivas de los profesionales de la salud.

\section{Profesionales de la salud, las ciencias sociales y la educación}

5. Las escuelas y los departamentos del gobierno responsables de la formación de los profesionales de la salud, las ciencias sociales y la educación deberán cerciorarse de que:

a) la formación de los profesionales de la salud, las ciencias sociales y la educación incluya el desarrollo de actitudes, conocimientos $\mathrm{y}$ habilidades para la creación de programas comunitarios de prevención y de promoción de la salud, apoyo comunitario, asesoramiento sobre modificaciones conductuales en pacientes, activismo social y presión política;

b) se proporcionen incentivos para apoyar la investigación sobre la transferencia de tecno- 
logías y asegurar el desarrollo, la evaluación y la difusión de métodos efectivos y baratos para prevenir y controlar los factores de riesgo cardiovascular en los planos colectivo e individual.

\section{Asociaciones y escuelas para profesionales de la salud y de la educación}

6. Las asociaciones y escuelas para profesionales de la salud y la educación que adiestran al personal de salud deben emprender las siguientes actividades:

a) Crear programas especiales para ayudar a los profesionales de la salud, líderes de la opinión pública, educadores, miembros de los medios publicitarios y políticos a adoptar estilos de vida saludables y a servir de modelos para la sociedad;

b) fomentar el abandono del tabaquismo entre sus miembros $\mathrm{y}$ luchar por la creación de ambientes libres de humo.

\section{Todos los grupos interesados en la difusión}

7. Los profesionales de la salud y la educación y sus instituciones, los organismos dedicados a la salud pública, los departamentos universitarios y las agencias gubernamentales apropiadas deberán formar alianzas para facilitar la transferencia de tecnologías y la difusión de políticas viables y de métodos de intervención validados por la práctica para fomentar la salud cardiovascular.

\section{Todos los profesionales de la salud}

8. Los profesionales de la salud deberán formar alianzas y grupos activistas para contrarrestar los intereses comerciales cuyas políticas, prácticas y productos sean perjudiciales para la salud cardiovascular.

\section{Los formuladores de políticas}

9. Los gobiernos de todos los niveles deberán tomar las siguientes medidas:

a) desarrollar y promover políticas para el fomento de la salud cardiovascular;

b) asumir la responsabilidad de proveer los recursos necesarios para coordinar las actividades en pro de la salud cardiovascular en los niveles nacional, local y comunitario; definir claramente las responsabilidades y funciones de cada nivel, y asegurar la instauración de infraestructuras y programas sostenibles.

\section{Las instituciones que financian la investigación}

10. Las agencias que financian proyectos de investigación deberán designar los recursos necesarios para las siguientes actividades:

a) desarrollar nuevos métodos y enfoques que faciliten la difusión y captación de los conocimientos existentes sobre prevención y de las intervenciones efectuadas por organizaciones dedicadas a la salud cardiovascular en todos los niveles;

b) realizar investigaciones en las organizaciones y estudios de evaluación para determinar el valor y costo de métodos alternativos para la creación, subvención y gestión de programas de salud cardiovascular, incluidas distintas opciones para su financiamiento por parte del sector privado;

c. aplicar sistemas para la vigilancia de los factores de riesgo de enfermedad cardiovascular, especialmente en poblaciones centinela, como la juventud y las personas que están pasando por cambios sociales y económicos acelerados;

d. aplicar sistemas para monitorear y notificar el progreso y los resultados de intervenciones planificadas y no planificadas.

\section{Agencias de fomento económico, social y sanitario}

11. Las organizaciones nacionales e internacionales de fomento económico, social y sanitario deberán emprender las siguientes acciones:

a. Reconocer el valor inherente a la inversión en la salud cardiovascular y proporcionar asistencia técnica y financiera a países beneficiarios y receptores de préstamos para que pongan en marcha, dentro del marco de sus planes de atención de salud y reforma social, iniciativas eficaces para la promoción de la salud, la atención clínica y la prevención de las enfermedades cardiovasculares, que sean efectivas en función del costo;

b. apoyar la difusión de información y la creación y mantenimiento de redes internacionales de profesionales de la salud, la educación y las ciencias sociales, y de organizaciones gubernamentales y no gubernamentales, a fin de conseguir el mutuo refuerzo de sus actividades y observar la evolución de los cambios experimentados.

\section{Comité para la Implementación de la Declaración de Victoria}

12. El Comité para la Implementación de la Declaración de Victoria deberá adoptar las siguientes medidas:

a. Convocar cuando sea oportuno, en colaboración con otras asociaciones científicas, conferencias y talleres internacionales, nacionales y locales sobre 
la salud cardiovascular para examinar los diferentes aspectos de la prevención desde el punto de vista de la formulación de políticas; apoyar la creación de alianzas para el fomento de la salud cardiovascular; brindar orientación sobre estrategias para la prevención de las enfermedades cardiovasculares en el contexto de la salud pública, y ayudar a difundirlas;

b. establecer vínculos nacionales e internacionales con las agencias de desarrollo económico, social y sanitario, a fin de facilitar el acceso a la asistencia técnica y financiera a los países en desarrollo y a los países desarrollados que están en proceso de adoptar economías de mercado.

Consejo Asesor de la Segunda Conferencia Internacional sobre la Salud Cardiovascular: Presidente: J.W. Farquhar, Facultad de Medicina, Uni- versidad de Stanford, Centro de Investigación para la Prevención de las Enfermedades, EUA. Presidentes adjuntos: H. Pardell, Departamento de Salud y Seguridad Social, Gobierno Autónomo de Cataluña, España; L. Komarek, Instituto Nacional de Salud Pública, República Checa. Miembros: V. Bales, Centros para el Control y la Prevención de Enfermedades, EUA; H. Blackburn, Universidad de Minnesota, EUA; R. Carmena, Universidad de Valencia, España; F. de Padua, Instituto Nacional de Cardiología Preventiva, Portugal; B. Finizola, Ascardio, Venezuela; H. Ghannem, Facultad de Medicina, Túnez; I. Glasunov, OMS, Dinamarca; F. Grande, Universidad de Zaragoza, España; L.W. Green, Universidad de Columbia Británica, Canadá; E. Greiser, Instituto de Bremen para la Prevención y la Medicina Social, Alemania; I.G. Gyarfas, OMS, Suiza; D.T. Jamison, Universidad de California en Los Ángeles, EUA; W.J. Keon, Asociación Cardiovascular del Canadá, Ottawa, Canadá; J.P. Koplan, The Prudential, EUA; T. Lasater, Programa de Salud del Corazón de Paw- tucket, EUA; R. Lessard, Consejo de Salud y Servicios Sociales de la Región de Montreal, Canadá; P. Lincoln, Dirección de Educación Sanitaria, Reino Unido; J. Mackay, Consultoría Asiática para el Control del Tabaco, Hong Kong; D. MacLean, Universidad de Dalhousie, Canadá; B. O'Connor, Junta de Salud de la Unión de North Shore, Canadá; R.G. Oganov, Centro Nacional de Medicina Preventiva, Rusia; P. Puska, Instituto Nacional de Salud Pública, Finlandia; D. Rae, WONCA, Canadá; K.S. Reddy, Instituto Nacional de Ciencias Médicas de la India; H. Restrepo, OPS, EUA; J.L. Rodicio, Universidad Autónoma de Madrid, España; S. Sans, Universidad Autónoma de Barcelona, España; M. Serrano, Universidad Autónoma de Madrid, España; S. Stachenko, Health Canada, Canadá; E. Stone, Instituto Nacional de Enfermedades Cardíacas, Pulmonares y Hemáticas, EUA; T. Strasser, Liga Mundial contra la Hipertensión, Suiza; J.R. Viskoper, Centro Médico Barzilai, Israel; Y. Yamori, Instituto Shimane de Ciencias de la Salud, Japón.

\section{REFERENCIAS}

Bandura A. Social foundations of thought and action: a social cognitive theory. Englewood Cliffs, New Jersey: Prentice Hall; 1986.

Chazova DV, Kalinina AM, Deev AD, Pavlova LI, Zyryaeva LA. The prevention of CHD in the population. En: Chazov EI, Oganov RG, eds. Prev Cardiol. Madison, Connecticut: International University Press; 1989:187-225.

Chicago Tribune. U.S. tobacco firms' overseas expansion generating smoke, heat. 20 de diciembre de 1993, sección comercial, p. 6.

Department de Sanitat i Seguretat Social de la Generalitat de Catalunya. Plan de salut de Catalunya 1993-1995. Barcelona: Department de Sanitat i Seguretat Social; 1993.

van Dis ISJ, van Assema P, Hellemans I. Educational tours in the supermarket. Trabajo presentado en la Segunda Conferencia Internacional sobre la Salud Cardiovascular, Barcelona, España, 28 de mayo al 1 de junio de 1995.

Erfurt JC, Foote AF, Heirich MA. The cost-effectiveness of work-site wellness programs for hypertension control, weight loss, and smoking cessation. J Occup Med 1991;33:962-970.

Farquhar JW. The case for dissemination research in health promotion and disease prevention.
En: Frankish CD, Green LW, Maclean D, Stachenko S, eds. Dissemination research in health promotion and disease prevention: proceedings of the Canadian Conference on Dissemination Research, Vancouver, Canadá, marzo de 1995. Vancouver: 1996.

Farquhar JW, Fortmann SP, Flora JA. Maccoby N. Methods of communication to influence behaviour. En: Holland WW, Detels R, Knox G, eds. Oxford Textbook of Public Health. New York: Oxford University Press; 1991:331-344.

Farquhar JW, Wood PD, Breitrose H, et al. Community education for cardiovascular health. Lancet 1977;1:1192-1195.

Forte JG, Pereira Miguel JM, Pereira Miguel MJ, de Padua F, Rose G. Salt and blood pressure: a community trial. J Hum Hypertens 1989;3: 179-184.

Green LW, Kreuter MW. Health promotion planning: an educational and environmental approach. Mountain View, California Mayfield Publishing Company; 1991.

Greiser E. Closing the social gap? Midterm results of the German Cardiovascular Prevention Study in Bremen City. Circulation 1992;85:868.

Max W, Rice D. Cost of smoking in California. Tobacco Control 1995;4(Supplement 1):S39-S46.
Narvaez M. Promoting a healthy lifestyle: the "Great Filipino Workout". Trabajo presentado en la Segunda Conferencia Internacional sobre la Salud Cardiovascular, Barcelona, España, 28 de mayo al 1 de junio de 1995.

Nester JM, Thieme JK. Strategies for structuring hospital and community health promotion and disease prevention education to reduce CVD. Trabajo presentado en la Segunda Conferencia Internacional sobre la Salud Cardiovascular, Barcelona, España, 28 de mayo al 1 de junio de 1995.

Parish R, Catford J, Nutbeam D. Breathing life into Wales: progress of the Welsh Heart Programme. Health Trends 1987;2:23-27.

Pelletier KR. A review and analysis of the health and cost-effective outcomes of studies of comprehensive health promotion and disease prevention programs at the worksite: 1991-1993 update. Am J Health Prom 1993;8:50-62.

Puska P, Salonen JT, Nissinen A, et al. Change in risk factors for coronary heart disease during 10 years of a community intervention programme (North Karelia project). Br Med J 1983;287: 1840-1844.

Roberts DCK, Clucas AC, Newton J. Development of an intervention strategy based on deep 
frying practices and frying oil use in a region with a high incidence of CHD. Trabajo presentado en la Segunda Conferencia Internacional sobre la Salud Cardiovascular, Barcelona, España, 28 de mayo a 1 de junio de 1995.

Salleras Ll, Rius E, Tresserras R, Vicente R. Working together for health gain at a regional level: the experience of Catalonia. Barcelona: Department of Health and Social Security of the Autonomous Government of Catalonia; 1994.

Stockley L. The promotion of healthier eating: a basis for action. London: The Health Education Authority; 1993.

Traynor MP, Glantz SA. The Development and Passage of Proposition 99, California's Tobacco Tax Initiative. San Francisco: University of California;
Institute for Policy Studies, School of Medicine; 1995.

Tresserras R, Serra-Majem L, Canela J, Armario P, Pardell H, Rue M, et al. Ecological association between hypertension and stroke in Catalonia (Spain): development and use of an ecological regression model. J Hum Hypertens 1990;4: 300-302.

U.S. Public Health Service. Healthy People 2000: midcourse review and 1995 revisions. Boston: Jones and Bartlett Publishers; 1995.

U.S. Public Health Service. Healthy People 2000: national health promotion and disease prevention objectives. Washington, DC: U.S. Department of Health and Human Services, Office of Disease Prevention and Health Promotion; 1991.
Vallbona C, Yusim S, Scherwitz L, Hennrikus D, Evans L. Advances in controlling hypertension in low-income patients. Am J Prev Med 1985;1:52-57.

Victoria Declaration on Heart Health. Victoria, Canada: Health and Welfare Canada; 1992.

de Vries MJ, de Vries M, Bokma J, van Dijk JP, Groothoff JW, Snijders H. The evaluation of a community project in the Netherlands. Trabajo presentado en la Segunda Conferencia Internacional sobre la Salud Cardiovascular, Barcelona, España, 28 de mayo al 1 de junio de 1995.

Warner K, Fulton G. The importance of tobacco to a country's economy: an appraisal of the tobacco industry's economic argument. Tobacco Control 1995;4:180-183.

\section{Congreso Latinoamericano de Nutrición}

\section{Fechas: $\quad 9$ a 15 de noviembre de 1997 \\ Lugar: $\quad$ Guatemala, Guatemala}

En las fechas arriba indicadas se celebrará en la ciudad de Guatemala el XI Congreso Latinoamericano de Nutrición titulado "Promoviendo la seguridad alimentaria y nutricional en América Latina". Este congreso es auspiciado por la Sociedad Latino Americana de Nutrición (SLAN) y un comité organizador local, y coordinado por el Instituto de Nutrición de Centro América y Panamá (INCAP). Los temas centrales propuestos para discusión son seguridad alimentaria y nutricional en el nivel local, economía alimentaria, tecnología y protección de recursos humanos, micronutrientes, nutrición de la mujer y los niños, dieta y salud en adultos, y vigilancia, monitoreo y evaluación de programas. El idioma oficial del congreso será el español, pero habrá algunas presentaciones en inglés.

\section{Información:}

Dr. Hernán L. Delgado, Presidente, SLAN y Director, INCAP Calzada Roosevelt Zona 11, Apartado postal 1118 Guatemala, Guatemala

HomePage Internet de INCAP en http://www.incap.org.gt Fax: (502) 473-6529, atención Dr. Hernán L. Delgado Correo electrónico: Hdelgado@incap.org.gt 